\title{
On some new matrix transformations
}

\author{
Rahmet Savaş ${ }^{1 *}$ and Ekrem Savaş²
}

${ }^{\text {*Correspondence: }}$ rahmet.savas@medeniyet.edu.tr

'Department of Mathematics, Medeniyet University, Istanbul, Turkey

Full list of author information is available at the end of the article

\begin{abstract}
In this paper, we characterize some matrix classes $\left(\omega(p, s), V_{\sigma}^{\lambda}\right),\left(\omega_{p}(s), V_{\sigma}^{\lambda}\right)$ and $\left(\omega_{p}(s), V_{\sigma}^{\lambda}\right)_{\text {reg }}$ under appropriate conditions.
\end{abstract}

\section{Introduction}

Let $w$ denote the set of all real and complex sequences $x=\left(x_{k}\right)$. By $l_{\infty}$ and $c$, we denote the Banach spaces of bounded and convergent sequences $x=\left(x_{k}\right)$ normed by $\|x\|=\sup _{k}\left|x_{k}\right|$, respectively. A linear functional $L$ on $l_{\infty}$ is said to be a Banach limit [1] if it has the following properties:

(1) $L(x) \geq 0$ if $n \geq 0$ (i.e., $x_{n} \geq 0$ for all $n$ ),

(2) $L(e)=1$, where $e=(1,1, \ldots)$,

(3) $L(D x)=L(x)$, where the shift operator $D$ is defined by $D\left(x_{n}\right)=\left\{x_{n+1}\right\}$.

Let $B$ be the set of all Banach limits on $l_{\infty}$. A sequence $x \in \ell_{\infty}$ is said to be almost convergent if all Banach limits of $x$ coincide. Let $\hat{c}$ denote the space of the almost convergent sequences. Lorentz [2] has shown that

$$
\hat{c}=\left\{x \in l_{\infty}: \lim _{m} d_{m, n}(x) \text { exists, uniformly in } n\right\},
$$

where

$$
d_{m, n}(x)=\frac{x_{n}+x_{n+1}+x_{n+2}+\cdots+x_{n+m}}{m+1} .
$$

The study of regular, conservative, coercive and multiplicative matrices is important in the theory of summability. In [3], King used the concept of the almost convergence of a sequence introduced by Lorentz to define more general classes of matrices than those of regular and conservative ones.

In [4], Schaefer defined the concepts of $\sigma$-conservative, $\sigma$-regular and $\sigma$-coercive matrices and characterized the matrix classes $\left(c, V_{\sigma}\right),\left(c, V_{\sigma}\right)_{\text {reg }}$ and $\left(l_{\infty}, V_{\sigma}\right)$, where $V_{\sigma}$ denotes the set of all bound sequences, all of whose invariant means (or $\sigma$-means) are equal. In [5], Mursaleen characterized the classes $\left(c(p), V_{\sigma}\right),\left(c(p), V_{\sigma}\right)_{\text {reg }}$ and $\left(l_{\infty}(p), V_{\sigma}\right)$ of matrices, which generalized the results due to Schaefer [4]. In [6], Mohiuddine and Aiyup defined the space $\omega(p, s)$ and obtained necessary and sufficient conditions to characterize the matrices of classes $\left(\omega(p, s), V_{\sigma}\right),\left(\omega_{p}(s), V_{\sigma}\right)$ and $\left(\omega_{p}(s), V_{\sigma}\right)_{\text {reg }}$.

Matrix transformations between sequence spaces have also been discussed by Savaş and Mursaleen [7], Basarir and Savaş [8], Mursaleen [5, 9-16], Vatan and Simsek [17], Savaş [18-24], Vatan [25] and many others.

\section{Springer}

(c) 2013 Savaş and Savaş; licensee Springer. This is an Open Access article distributed under the terms of the Creative Commons Attribution License (http://creativecommons.org/licenses/by/2.0), which permits unrestricted use, distribution, and reproduction in any medium, provided the original work is properly cited. 
In this paper we characterize the matrix classes from this space to the space $V_{\sigma}^{\lambda}$, i.e., we obtain necessary and sufficient conditions to characterize the matrices of classes $\left(\omega(p, s), V_{\sigma}^{\lambda}\right),\left(\omega_{p}(s), V_{\sigma}^{\lambda}\right)$ and $\left(\omega_{p}(s), V_{\sigma}^{\lambda}\right)_{\mathrm{reg}}$.

\section{Preliminaries}

Let $\sigma$ be a one-to-one mapping from the set $N$ of natural numbers into itself. A continuous linear functional $\varphi$ on $l_{\infty}$ is said to be an invariant mean or $\sigma$-mean if and only if

(i) $\varphi(x) \geq 0$ when the sequence $x=\left(x_{k}\right)$ has $x_{k} \geq 0$ for all $k$;

(ii) $\varphi(e)=1$;

(iii) $\varphi(x)=\varphi\left(x_{\sigma(k)}\right)$ for all $x \in l_{\infty}$.

Let $V_{\sigma}$ denote the set of bounded sequences all of whose $\sigma$-means are equal. We say that a sequence $x=\left(x_{k}\right)$ is $\sigma$-convergent if and only if $x \in V_{\sigma}$. For $\sigma(n)=n+1$, the set $V_{\sigma}$ is reduced to the set $\hat{c}$ of almost convergent sequences $[2,26]$.

If $x=\left(x_{n}\right)$, write $T x=\left(x_{\sigma(n)}\right)$. It is easy to show that

$$
V_{\sigma}=\left\{x \in l_{\infty}: \lim _{m} t_{m n}(x)=L \text {, uniformly in } n ; L=\sigma-\lim x\right\},
$$

where

$$
t_{m n}(x)=\frac{1}{m+1} \sum_{j=0}^{m} T^{j} x_{n}
$$

and $\sigma^{m}(n)$ denotes the $m$ th iterate of $\sigma$ at $n$.

If $p_{k}$ is real and positive, we define (see Maddox [27])

$$
c_{0}(p)=\left\{x: \lim _{k \rightarrow \infty}\left|x_{k}\right|^{p_{k}}=0\right\}
$$

and

$$
c(p)=\left\{x: \lim _{k \rightarrow \infty}\left|x_{k}-l\right|^{p_{k}}=0 \text { for some } l\right\} .
$$

The classes $\left(\omega(p, s), V_{\sigma}\right),\left(\omega_{p}(s), V_{\sigma}\right)$ and $\left(\omega_{p}(s), V_{\sigma}\right)_{\text {reg }}$ have been defined by Mohiuddine and Aiyup [6] and, for $p=\left(p_{k}\right)$ with $p_{k}>0$, the space $\omega(p, s)$ is defined for $s \geq 0$ by

$$
\omega(p, s)=\left\{x: \frac{1}{n} \sum_{k=1}^{n} k^{-s}\left|x_{k}-l\right|^{p_{k}} \rightarrow 0, n \rightarrow \infty \text { for some } l, s \geq 0\right\},
$$

where $s=\left(s_{k}\right)$ is an arbitrary sequence with $s_{k} \neq 0(k=1,2, \ldots)$. If $p_{k}=p$, for each $k$, we have $\omega(p, s)=\omega_{p}(s)$.

The sequence space

$$
\omega(p)=\left\{x: \frac{1}{n} \sum_{k=1}^{n}\left|x_{k}-l\right|^{p_{k}} \rightarrow 0, n \rightarrow \infty\right\}
$$

for some $l$, which has been investigated by Maddox is the special case of $\omega(p, s)$ which corresponds to $s=0$. Obviously $\omega(p) \subset \omega(p, s)$. 
We further define the following.

Let $\lambda=\left(\lambda_{m}\right)$ be a non-decreasing sequence of positive numbers tending to $\infty$ such that

$$
\lambda_{m+1} \leq \lambda_{m}+1, \quad \lambda_{1}=1 .
$$

A sequence $x=\left(x_{k}\right)$ of real numbers is said to be $(\sigma, \lambda)$-convergent to a number $L$ if and only if $x \in V_{\sigma}^{\lambda}$, where

$$
\begin{aligned}
& V_{\sigma}^{\lambda}=\left\{x \in l_{\infty}: \lim _{m \rightarrow \infty} t_{m n}(x)=L, \text { uniformly in } n ; L=(\sigma, \lambda)-\lim x\right\}, \\
& t_{m n}(x)=\frac{1}{\lambda_{m}} \sum_{i \in I_{m}} x_{\sigma^{i}(n)},
\end{aligned}
$$

and $I_{m}=\left[m-\lambda_{m}+1, m\right]$. Note that $c \subset V_{\sigma}^{\lambda} \subset l_{\infty}$. For $\sigma(n)=n+1, V_{\sigma}^{\lambda}$ reduces to the space $\hat{V}_{\lambda}$ of almost $\lambda$-convergent sequences [28]; and if we take $\sigma(n)=n+1$ and $\lambda_{m}=m$, then $V_{\sigma}^{\lambda}$ reduces to $\hat{c}$ (see [29]). Further, if we take $\lambda_{m}=m$, then $V_{\sigma}^{\lambda}$ reduces to $V_{\sigma}$.

If $E$ is a subset of $\omega$, then we write $E^{+}$for a generalized Köthe-Toeplitz dual of $E$; i.e.,

$$
E^{+}=\left\{a: \sum_{k} a_{k} x_{k} \text { converges for every } x \in E\right\}
$$

If $0<p_{k} \leq 1$, then $\omega^{+}(p)=\mathbb{M}$, where

$$
\mathbb{M}=\left\{a: \sum_{r=0}^{\infty} \max _{r}\left\{\left(2^{r} \cdot N^{-1}\right)^{\frac{1}{p_{k}}}\left|a_{k}\right|\right\}<\infty \text { for some integer } N>1\right\},
$$

and max is the maximum taken over $2^{r} \leq k<2^{r+1}$ (see Theorem 4, [30]).

If $X$ is a topological linear space, we denote by $X^{*}$ the continuous dual of $X$; i.e., the set of all continuous linear functionals on $X$. Obviously,

$$
[\omega(p, s)]^{*}=\left\{a: \sum_{r=0}^{\infty} \max _{r}\left\{\left(2^{r} \cdot N^{-1}\right)^{\frac{1}{p_{k}}}\left|\frac{a_{k}}{s_{k}}\right|\right\}<\infty \text { for some integer } N>1\right\} .
$$

\section{Main results}

Let $X$ and $Y$ be two nonempty subsets of the space $w$ of complex sequences. Let $A=\left(a_{n k}\right)$ $(n, k=1,2, \ldots)$ be an infinite matrix of complex numbers. We write $A x=\left(A_{n}(x)\right)$ if $A_{n}(x):=$ $\sum_{k} a_{n k} x_{k}$ converges for each $n$. (Throughout, $\sum_{k}$ will denote summation over $k$ from $k=1$ to $k=\infty$.) If $x=\left(x_{k}\right) \in X$ implies that $A x=\left(A_{n}(x)\right) \in Y$, we say that $A$ defines a (matrix) transformation from $X$ to $Y$ and we denote it by $A: X \rightarrow Y$. By $(X, Y)$ we mean the class of matrices $A$ such that $A: X \rightarrow Y$.

We now characterize the matrices in the class $\left(c_{0}(p), V_{\sigma_{0}}^{\lambda}(p)\right)$. We write

$$
t_{m, n}(A x)=\sum_{k} a(n, k, m) x_{k}
$$

where

$$
a(n, k, m)=\frac{1}{\lambda_{m}} \sum_{i \in I_{m}} a_{\sigma^{i}(n), k} .
$$


Theorem 3.1 Let $0<p_{k} \leq 1$, then $A \in\left(\omega(p, s), V_{\sigma}^{\lambda}\right)$ if and only if

(i) there exists an integer $B>1$ such that for every $n$

$$
D_{n}=\sup _{m} \sum_{r=0}^{\infty} \max _{r}\left(2^{r} \cdot B^{-1}\right)^{\frac{1}{p_{k}}}\left|\frac{a(n, k, m)}{s_{k}}\right|<\infty
$$

(ii) $\alpha_{(k)}=\left\{a_{n k}\right\}_{n=1}^{\infty} \in V_{\sigma}^{\lambda}$ for each $k$;

(iii) $\alpha=\left\{\sum_{k} a_{n k}\right\}_{n=1}^{\infty} \in V_{\sigma}^{\lambda}$.

In this case the $\sigma-\lim$ of $A x$ is $(\lim x)\left[u-\sum_{k} u_{k}\right]+\sum_{k} u_{k} x_{k}$ for every $x \in w(p, s)$, where $u=\sigma-\lim a$ and $u_{k}=\sigma-\lim a_{(k)}, k=1,2, \ldots$

Proof Suppose that $A \in\left(\omega(p, s), V_{\sigma}^{\lambda}\right)$. Define $e^{k}=(0,0, \ldots, 1,0, \ldots)$ having 1 in the $k$ th coordinate sequence. Since $e$ and $e^{k}$ are in $\omega(p, s)$, necessity of (ii) and (iii) is clear. We know that $\sum_{k} a(n, k, m) x_{k}$ converges for each $m, n$ and $x \in \omega(p, s)$. Therefore $(a(n, k, m))_{k} \in \omega^{+}(p, s)$ and

$$
\sum_{r=0}^{\infty} \max _{r}\left(2^{r} \cdot B^{-1}\right)^{\frac{1}{p_{k}}}\left|\frac{a(n, k, m)}{s_{k}}\right|<\infty
$$

for each $m, n$ (see [31]). Furthermore, if $f_{m n}(x)=t_{m n}(A x)$, then $\left\{f_{m n}\right\}_{m}$ is a sequence of continuous linear functionals on $\omega(p, s)$ such that $\lim _{m} t_{m n}(A x)$ exists. Therefore, by using the Banach-Steinhaus theorem, the necessity of (i) follows immediately.

Conversely, suppose that the conditions (i), (ii) and (iii) hold and $x \in \omega(p, s)$. We know that $(a(n, k, m))_{k}$ and $u_{k}$ are in $\omega^{+}(p, s)$ and that the series $\sum_{k} a(n, k, m) x_{k}$ and $\sum_{k} u_{k} x_{k}$ converge for each $m, n$. Write

$$
c(n, k, m)=a(n, k, m)-u_{k} .
$$

Then

$$
\sum_{k} a(n, k, m) x_{k}=\sum_{k} u_{k} x_{k}+l \sum_{k} c(n, k, m)+\sum_{k} c(n, k, m)\left(x_{k}-l\right)
$$

by (ii) for some integer $k_{0}>0$, we have

$$
\lim _{m} \sum_{k \leq k_{0}} c(n, k, m)\left(x_{k}-l\right)=0, \quad \text { uniformly in } n,
$$

where $l$ is the limit of $x$ for $x \in \omega(p, s)$. Since

$$
\begin{aligned}
& \sup _{m, n} \sum_{r} \max _{r}\left(2^{r} \cdot B^{-1}\right)^{\frac{1}{p_{k}}}|c(n, k, m)| \leq 2 D_{n}, \\
& \lim _{m} \sum_{k \leq k_{0}}\left|\frac{a(n, k, m)-u_{k}}{s_{k}}\right|\left|s_{k}\left(x_{k}-l\right)\right|=0,
\end{aligned}
$$

uniformly in $n$, whence

$$
\lim _{n} \sum_{k} a(n, k, m) x_{k}-l \cdot u+\sum_{k} u_{k}\left(x_{k}-l\right) .
$$


Theorem 3.2 Let $1 \leq p_{k}<\infty$, then $A \in\left(\omega_{p}(s), V_{\sigma}^{\lambda}\right)$ if and only if

(i) for every $n$

$$
M(A)=\sup _{m} \sum_{r} 2^{\frac{r}{p}}\left(\sum_{r}\left|\frac{a(n, k, m)}{s_{k}}\right|^{q}\right)^{\frac{1}{q}}<\infty,
$$

where $p^{-1}+q^{-1}=1$

(ii) $\alpha_{(k)} \in V_{\sigma}^{\lambda}$ for each $k$;

(iii) $\alpha \in V_{\sigma}^{\lambda}$.

Proof Assume that the conditions are satisfied and let $x \in \omega_{p}(s)$. Then

$$
\left|t_{m n}(A x)\right| \leq \sum_{r=0}^{\infty} \sum_{r}\left|\frac{a(n, k, m) s_{k} x_{k}}{s_{k}}\right| \leq \sum_{r=0}^{\infty}\left(\sum_{r}\left|\frac{a(n, k, m)}{s_{k}}\right|^{q}\right)^{\frac{1}{q}} \cdot\left(\sum_{r}\left|x_{k}\right|^{p}\right)^{\frac{1}{p}},
$$

and hence $t_{m n}(A x)$ is absolutely and uniformly convergent for each $m, n$. Note that (i) and (ii) imply that

$$
\sum_{r=0}^{\infty} 2^{\frac{r}{p}}\left(\sum_{r}\left|s_{k} u_{k}\right|\right)^{\frac{1}{q}} \leq M(A)<\infty
$$

so that by Hölder's inequality, $\sum_{k} u_{k} x_{k}<\infty$. Now as in the converse part of Theorem 3.1, it follows that $A \in\left(\omega_{p}(s), V_{\sigma}^{\lambda}\right)$.

Conversely, suppose that $A \in\left(\omega_{p}(s), V_{\sigma}^{\lambda}\right)$. Since $e^{k}$ and $e$ are in $\omega_{p}(s)$, the necessity of (ii) and (iii) is clear. For the necessity of (i), suppose that

$$
t_{m n}(A x)=\sum_{k} a(n, k, m) x_{k}
$$

exits for each $n$ whenever $x \in \omega_{p}(s)$. Then, for each $n$ and $r \geq 0$, write

$$
f_{n r}(x)=\sum_{r} a(n, k, m) x_{k}
$$

Then $\left\{f_{n r}\right\}_{m}$ is a sequence of continuous linear functionals on $\omega_{p}(s)$. Since

$$
\left|f_{n r}(x)\right| \leq\left(\sum_{r}\left|\frac{a(n, k, m)}{s_{k}}\right|^{q}\right)^{\frac{1}{q}} \cdot\left(\sum_{r}\left|s_{k} \cdot x_{k}\right|^{p}\right)^{\frac{1}{p}} \leq 2^{\frac{r}{p}}\left(\sum_{r}\left|\frac{a(n, k, m)}{s_{k}}\right|^{q}\right)^{\frac{1}{q}} \cdot\|x\|,
$$

it follows that for each $n$,

$$
\lim _{j} \sum_{r=0}^{j} f_{m r}(x)=t_{m n}(A x)
$$

is in the dual space $\omega_{p}^{*}$. Hence there exists a $K_{m n}$ such that

$$
\left|\frac{a(n, k, m)}{s_{k}}\right| \leq K_{m n}\|x\|
$$


For each $n$ and any integer $j>0$, define $x \in \omega_{p}(s)$ as in [30] (Theorem 7, p.173), we get

$$
\sum_{r=0}^{j} 2^{\frac{r}{p}}\left(\sum_{r}\left|\frac{a(n, k, m)}{s_{k}}\right|^{q}\right)^{\frac{1}{q}} \leq K_{m n} .
$$

Hence, for each $n$,

$$
\sum_{r=0}^{\infty} 2^{\frac{r}{p}}\left(\sum_{r}\left|\frac{a(n, k, m)}{s_{k}}\right|^{q}\right)^{\frac{1}{q}} \leq K_{m n}<\infty .
$$

Since $t_{m n}(A x)$ is absolutely convergent, we get

$$
\left|t_{m n}(A x)\right| \leq \sum_{r=0}^{\infty} 2^{\frac{r}{p}}\left(\sum_{r}\left|\frac{a(n, k, m)}{s_{k}}\right|^{q}\right)^{\frac{1}{q}}\|x\|,
$$

so that

$$
K_{m n} \leq \sum_{r=0}^{\infty} 2^{\frac{r}{p}}\left(\sum_{r}\left|\frac{a(n, k, m)}{s_{k}}\right|^{q}\right)^{\frac{1}{q}} .
$$

By virtue of (3.2) and (3.3),

$$
K_{m n}=\sum_{r=0}^{\infty} 2^{\frac{r}{p}}\left(\sum_{r}\left|\frac{a(n, k, m)}{s_{k}}\right|^{q}\right)^{\frac{1}{q}} .
$$

Finally, by (Theorem 11, [30], p.114) for every $n$, the existence of $\lim _{m} t_{m n}(A x)$ on $\omega_{p}(s)$ implies that

$$
\sup _{m} K_{m n}=\sup _{m} \sum_{r=0}^{\infty} 2^{\frac{r}{p}}\left(\sum_{r}\left|\frac{a(n, k, m)}{s_{k}}\right|^{q}\right)^{\frac{1}{q}}<\infty,
$$

which is (i).

Theorem 3.3 Let $0<p_{k}<\infty$, then $A \in\left(\omega_{p}(s), V_{\sigma}\right)_{\text {reg }}$ if and only if conditions (i), (ii) with $\sigma-\lim =0$ and (iii) with $\sigma-\lim =+1$ of Theorem 3.2 hold.

\section{Competing interests}

The authors declare that they have no competing interests.

\section{Authors' contributions}

Both authors completed the paper together. Both authors read and approved the final manuscript.

\section{Author details}

${ }^{1}$ Department of Mathematics, Medeniyet University, Istanbul, Turkey. ${ }^{2}$ Department of Mathematics, Istanbul Ticaret University, Uskudar, Istanbul, Turkey.

\section{Acknowledgements}

Dedicated to Professor Hari M Srivastava.

We would like to thank the referees for their valuable comments.

Received: 21 November 2012 Accepted: 18 April 2013 Published: 20 May 2013 


\section{References}

1. Banach, S: Theorie des Operations lineaires. Instytut Matematyczny Polskiej Akademi Nauk, Warsaw (1932)

2. Lorentz, GG: A contribution to the theory of divergent sequences. Acta Math. 80, 167-190 (1948)

3. King, JP: Almost summable sequences. Proc. Am. Math. Soc. 17, 1219-1258 (1966)

4. Schaefer, P: Infinite matrices and invariant means. Proc. Am. Math. Soc. 36, 104-110 (1972)

5. Mursaleen, M: Invariant means and some matrix transformations. Tamkang J. Math. 10, 181-184 (1979)

6. Mohiuddine, SA, Aiyup, M: Matrix transformations of strongly convergent sequences into $V_{\sigma}$. Filomat 24(3), 103-109 (2010)

7. Savaş, E, Mursaleen, M: Matrix transformations in some sequence spaces. Istanbul Univ. Fen Fak. Mat. Derg. 52, 1-5 (1993)

8. Başarir, M, Savaş, E: On matrix transformations of some generalized sequence space. Math. Slovaca 45(2), 155-162 (1995)

9. Mursaleen, M: On some new invariant matrix methods of summability. Q. J. Math. 34, 77-86 (1983)

10. Mursaleen, M, Jarrah, AM, Mohiuddine, SA: Bounded linear operators for some new matrix transformations. Iran. J. Sci. Technol., Trans. A, Sci. 33(A2), 169-177 (2010)

11. Mursaleen, M: On infinite matrices and invariant means. Indian J. Pure Appl. Math. 10(4), 457-460 (1979)

12. Mursaleen, M, Gaur, AK, Chishti, TA: On some new sequence spaces of invariant means. Acta Math. Hung. 75, 185-190 (1997)

13. Mursaleen, M, Savaş, E, Aiyub, M, Mohiuddine, SA: Matrix transformations between the spaces of Cesaro sequences and invariant means. Int. J. Math. Math. Sci. 200, Article ID 74319 (2006)

14. Mursaleen, M: Some matrix transformations on sequence spaces of invariant means. Hacet. J. Math. Stat. 38(3), 259-264 (2009)

15. Mursaleen, M: On A-invariant mean and A-almost convergence. Anal. Math. 37(3), 173-180 (2011)

16. Mursaleen, M, Mohiuddine, SA: Some matrix transformations of convex and paranormed sequence spaces into the spaces of invariant means. J. Funct. Spaces Appl. 2012, Article ID 612671 (2012)

17. Karakaya, V, Simsek, N: On some matrix transformations. Int. Math. J. 4(1), 19-25 (2003)

18. Savaş, E: On infinite matrices and lacunary $\sigma$-convergence. Appl. Math. Comput. 218(3), 1036-1040 (2011)

19. Savaş, E: Matrix transformations of some generalized sequence spaces. J. Orissa Math. Soc. 4(1), 37-51 (1985)

20. Savaş, E: Matrix transformations and absolute almost convergence. Bull. Inst. Math. Acad. Sin. 15(3), $345-355$ (1987)

21. Savaş, E: Matrix transformations between some new sequence spaces. Tamkang J. Math. 19(4), $75-80$ (1988)

22. Savaş, E: $\sigma$-summable sequences and matrix transformations. Chin. J. Math. 18(3), 201-207 (1990)

23. Savaş, E: Matrix transformations and almost convergence. Math. Stud. 59(1-4), 170-176 (1991)

24. Savaş, E: Matrix transformations of $X_{p}$ into $C_{s}$. Punjab Univ. J. Math. 24, 59-66 (1991)

25. Karakaya, $\vee: \theta_{\sigma}$-summable sequences and some matrix transformations. Tamkang J. Math. 35(4), $313-320$ (2004)

26. Qamaruddin, Mohiuddine, SA: Almost convergence and some matrix transformations. Filomat 21(2), 261-266 (2007)

27. Maddox, IJ: Spaces of strongly summable sequences. Q. J. Math. 18, 345-355 (1967)

28. Savaş, E: Strongly almost convergence and almost $\lambda$-statistical convergence. Hokkaido Math. J. 29, 63-68 (2000)

29. Nanda, S: Infinite matrices and almost convergence. J. Indian Math. Soc. 40, 173-184 (1976)

30. Maddox, IJ: Elements of Functional Analysis. Cambridge University Press, Cambridge (1970)

31. Lascarides, CG, Maddox, IJ: Matrix transformations between some classes of sequences. Proc. Camb. Philos. Soc. 68 99-104 (1970)

doi:10.1186/1029-242X-2013-254

Cite this article as: Savaş and Savaş: On some new matrix transformations. Journal of Inequalities and Applications 2013 2013:254.

\section{Submit your manuscript to a SpringerOpen ${ }^{\circ}$ journal and benefit from:}

- Convenient online submission

- Rigorous peer review

- Immediate publication on acceptance

- Open access: articles freely available online

- High visibility within the field

- Retaining the copyright to your article 